\title{
Propos d'une historienne des femmes et du genre
}

Thoughts from an Historian in Women's and Gender Studies

\section{Françoise Thébaud}

\section{OpenEdition}

Journals

Édition électronique

URL : http://journals.openedition.org/questionsdecommunication/567

DOI : 10.4000/questionsdecommunication.567

ISSN : 2259-8901

\section{Éditeur}

Presses universitaires de Lorraine

\section{Édition imprimée}

Date de publication : 1 juillet 2009

Pagination : 221-245

ISBN : 978-2-86480-989-0

ISSN : 1633-5961

\section{Référence électronique}

Françoise Thébaud, "Propos d'une historienne des femmes et du genre », Questions de communication [En ligne], 15 | 2009, mis en ligne le 01 août 2011, consulté le 03 mai 2019. URL : http:// journals.openedition.org/questionsdecommunication/567; DOI : 10.4000/ questionsdecommunication.567 


\title{
> ÉCHANGES
}

FRANÇOISE THÉBAUD

Laboratoire d'histoire d'Avignon

Université d'Avignon et des pays de Vaucluse

francoise.thebaud@univ-avignon.fr

\section{PROPOS D'UNE HISTORIENNE DES FEMMES ET DU GENRE}

\begin{abstract}
Résumé.-Y a-t-il un gender turn en sciences humaines? Réponse à la contribution de Marlène Coulomb-Gully, cet article présente le cas de la discipline historique en France, discipline ancienne et traditionnellement masculine. Dans un parcours chronologique de plus de trente-cinq ans, il expose les conditions de production et de réception de l'histoire des femmes et du genre : contexte intellectuel et politique, trajectoires de ses promotrices, politiques académiques et éditoriales, débats historiographiques... La comparaison avec les sciences de l'information et de la communication met en lumière les réticences françaises aux approches de genre et les paradoxes de la situation actuelle. Elle souligne aussi que le genre ne peut pas être considéré seulement comme un concept importé des États-Unis et que ses usages sont multiples et féconds.
\end{abstract}

Mots clés. - Histoire des femmes, genre, historiographie, politiques académiques, spécificités disciplinaires. 
e souhaite d'abord remercier Marlène Coulomb-Gully (2009) pour sa précieuse contribution sur les relations entretenues entre les sciences de l'information et de la communication (SIC) et les études genre. Cette contribution, qui représente un exigeant travail de synthèse et de médiation, appelle à un gender turn dans cette discipline en France et défend la fécondité de l'approche de genre pour les SIC. Passeuse et pionnière avec quelques autres cité-e-s au fil de l'article, Marlène Coulomb-Gully brosse d'abord, à l'usage de ses collègues, un bref historique de «la difficile implantation des études de genre en France ». Puis, elle établit un bilan nuancé d'une « reconnaissance tardive » et tente d'expliquer à la fois la « marginalité de l'approche genrée en $\mathrm{SIC}$ » et le « virage pris à l'orée du $3^{\mathrm{e}}$ millénaire ». Enfin, et avec optimisme, elle souligne l'existence de « convergences de fond entre approche communicationnelle et genre » et montre, à travers l'analyse de quelques travaux anglophones et français, les potentialités de la rencontre pour l'étude des médias, de la communication politique et interpersonnelle, des nouvelles technologies ou d'autres objets encore. Par ailleurs, la lecture de cet article sera très stimulante pour les chercheuses et chercheurs d'autres disciplines (sociologie, histoire, science politique ou sciences du langage, psychologie) qui y découvriront des travaux sur des questions vives du très contemporain et une invitation à l'interdisciplinarité qui a longtemps marqué et caractérisé les recherches sur les femmes et le genre.

Sans doute parce que je me suis livrée au même exercice de bilan et de transmission pour ce qui concerne l'histoire des femmes et du genre', il m'a été demandé de réagir à la contribution de Marlène CoulombGully et de dire ce qu'il en est de la rencontre entre genre et histoire. J'ai alors proposé au comité de rédaction de Questions de communication de publier l'article ci-dessous que je venais de rédiger pour un ouvrage franco-québécois ${ }^{2}$ et qui répondait, me semble-t-il, à la demande. Je lui joins ces quelques pages introductives qui mettent en perspective, par la comparaison, les deux contributions et apportent aux lecteurs de la revue le regard extérieur d'une discipline plus ancienne que les SIc.

\footnotetext{
Voir Thébaud (2007) et, du même auteure, les références indiquées dans la présente contribution.

2 Cet article, titré « Politiques du genre en sciences humaines. L'exemple de la discipline historique en France », a été rédigé pour l'ouvrage suivant : A. Duchêne, Cl. Moïse, dirs, Langage, genre et sexualité, Montréal, Éd. Nota Bene, 2009. Je remercie vivement les éditeurs du volume et la maison d'édition de m'avoir autorisée à le reprendre ici. Questions de communication a modifié le titre et adapté la première phrase. La revue a aussi présenté les références conformément à sa maquette (voir la remarque finale des pages introductives).
} 
Les savoirs ont une histoire qui n'est pas seulement celle de l'accumulation des connaissances, mais aussi celle de leurs conditions de production et de réception, conditions marquées par des rapports de pouvoir institutionnels et humains. Dans la discipline historique comme dans les SIc, l'approche de genre a longtemps été frappée - et l'est encore partiellement - d'un soupçon d'illégitimité et de non-scientificité, ses détracteurs mobilisant dans la controverse des formes d'anti-américanisme. Discipline jeune, les sIc ont, dans leur recherche de respectabilité, évité les questions importées et peu légitimes. Mais les 35 à 40 ans d'histoire des femmes et du genre en France permettent, me semble-t-il, de nuancer le caractère importé des études de genre et de réviser, sur quelques points, le récit chronologique présenté par Marlène Coulomb-Gully à partir de synthèses européennes où l'histoire est peu présente.

Certes, en histoire comme pour les SIC, les chercheuses et chercheurs américains ont été pionniers, chronologiquement et par l'ampleur de leur production scientifique. Mais, l'approche de genre dans la discipline historique est autant le résultat de débats internes à l'histoire des femmes que du transfert d'un concept américain promu par Joan Scott qui l'a acclimaté à sa discipline dans la deuxième moitié des années $80^{3}$. Son article paru en 1986 dans la prestigieuse American Historical Review fut rapidement - fait exceptionnel - traduit en français par Les Cahiers du GRIF, qui ont accueilli en 1988 le dossier présenté par Christine Planté, Michèle Riot-Sarcey et Eleni Varikas, mais avaient peu de lecteurs dans la communauté des historiens ${ }^{4}$.

Par ailleurs, dans une discipline fondamentalement empirique, l'histoire des femmes et du genre, qui s'est toujours interrogée sur les modalités de son écriture, a été et reste un laboratoire de réflexion méthodologique et épistémologique. Elle constitue une des spécialités les plus théoriciennes - parfois critiquée comme telle -, le genre ayant aussi été un des moyens de s'imposer à la discipline et de la transformer. Comme l'écrivait Françoise Collin (1988: 5) en introduction de la livraison citée des Cahiers du GRIF, il s'agissait de « dégager les recherches féministes [d'une identification aux recherches sur les femmes] et de faire apparaître leur caractère opératoire sur l'ensemble du champ du savoir ».

3 Toute circulation d'idées s'accompagne de phénomènes d'appropriation, sélection, réélaboration. Signalons à cet égard que la French feminist theory (longtemps représentée par L. Irigaray, H. Cixous, J. Kristeva) fut une invention américaine qui ne correspondait pas à la réalité diverse et conflictuelle des féminismes français et qu'associer sous ce terme L. Irigaray et S. de Beauvoir est inexact (voir Nouvelles questions féministes, 1996). II est vrai cependant que l'ouvrage de D. Cavallaro (2003) est sous ce titre beaucoup moins exclusif. 4 Revue fondée par la philosophe Fr. Collin, Les cahiers du GRIF ne sont pas lus pas les historiens/nes, en dehors de celles qui travaillent sur les femmes et le genre. 
Si l'approche féministe - qualification peu utilisée par les historiennes - a en histoire comme ailleurs revendiqué « un lien fort avec le terrain et le concret »-créant de nouveaux objets historiques (corps, maternité, sexualité, féminismes, etc.) et suscitant de nouvelles pratiques comme I'histoire orale ${ }^{5}$-, elle n'y a jamais négligé la réflexion théorique. Présenter les études de genre comme « l'émanation directe » de «l'Amérique des religions et des corps » n'est qu'une tendance actuelle de journalistes français qui, ne connaissant pas les travaux antérieurs, considèrent les gender studies comme un domaine neuf et l'assimilent aux recherches récentes en histoire et sociologie des sexualités et des identités. Malgré l'intérêt incontestable de ces recherches, le propos - réducteur - doit être contré car il rend invisibles et délégitime d'autres thématiques qui constituent aussi l'histoire du genre.

Enfin, il est possible de complexifier la chronologie proposée. II serait en effet intéressant d'engager des recherches approfondies sur le début de reconnaissance des années 80 qui m'apparaît, pour diverses raisons, comme une occasion manquée d'institutionnaliser les études femmes et genre en France, un contexte aussi favorable ne s'étant jamais retrouvé6. Le creux des années 90 est alors relativisé, Christine Bard (2003) ayant même qualifié d'embellie la période 1995-2002 où de nombreuses initiatives de personnes et d'associations tentent de pallier le déficit institutionnel. À l'historienne aussi paraît remarquable l'émergence en science politique des problématiques de genre qui s'affirment, grâce à une jeune génération, dans plusieurs thèses et publications récentes bien accueillies par la discipline ${ }^{7}$. Et en histoire comme ailleurs, la nouvelle donne du $3^{\mathrm{e}}$ millénaire est marquée par l'utilisation de plus en plus fréquente du terme « genre » qui fut plus tardive que celle du concept. Mais, comme le souligne Joan Scott cité par Marlène Coulomb-Gully, cela se fait bien souvent au risque de l'affadissement d'un synonyme de " femmes, de sexes, de rôles sexués, renaturalisé et non dénaturalisant ». J'ai également plusieurs fois souligné que la reconnaissance intellectuelle de l'histoire des femmes et du genre - partielle mais incontestable - n'était pas suivie d'une reconnaissance institutionnelle (postes fléchés, laboratoires de recherche) qui permet à un domaine de recherche de développer toutes ses potentialités et d'intégrer les formes habituelles du savoir transmis.

\footnotetext{
5 Sur les convergences entre histoire des femmes et histoire orale, voirThébaud, Dermenjian (2009).

6 Dans mon analyse de la politique menée par le ministère des Droits de la Femme entre 198I et 1986 (Thébaud, 200 I), je n'ai qu'effleuré ce point.

7 Signalons le manuel de la collection « Ouvertures politiques 》 aux éditions De Boeck (Bereni et al., 2008).
} 
Gender turn? C'est autour de l'usage de ce terme par Marlène CoulombGully et de sa signification que portera un deuxième ensemble de remarques. L'article écrit est certes le signe d'un tournant qu'il contribuera sans doute à accentuer. Mais le tournant des SIC reste en France tout relatif, malgré un taux élevé de féminisation de son corps d'enseignantschercheurs 8 . À suivre son auteure pourtant optimiste, les enseignements spécialisés sont quasi absents de l'offre universitaire - 12 heures par an dans un seul établissement au niveau bac +5 ! - , ce qui n'empêche pas l'intégration dans des cours généraux de séances ou de thèmes « genre », pratique plus fréquente en histoire que l'uv fléchée comme telle qui attire les étudiants mais indispose maints collègues. Du côté des recherches publiées dans l'ensemble des revues depuis dix ans, le bilan peut paraître maigre aussi : dix numéros thématiques consacrés aux femmes ou au genre, soit par rapide comptage, moins de 2,5\% de l'ensemble?

Les titres de ces numéros thématiques que liste en annexe de sa contribution Marlène Coulomb-Gully me suggèrent une autre remarque. « Sexe », « genre », « féminin », « sexué », « femmes » : la diversité des appellations témoigne de la diversité des approches ou des mots pour les dire, diversité qu'il est nécessaire de reconnaître et de faire dialoguer. En dehors des effets de mode ou d'appel aux subventions européennes pour lesquelles le genre est incontournable, l'approche de genre et l'usage du terme ne s'imposent pas facilement. En histoire, ils se sont construits peu à peu à partir de questionnements de plus en plus complexes : où sont et que font les femmes? Femmes et hommes ont-ils la même place? Qui définit et comment la différence des sexes? Avec quels effets de pouvoir? Le genre précède-t-il le sexe ? Comment rendre compte de l'absence de recouvrement sexe/genre? Dans quelle tradition culturelle s'inscrivent le concept et le terme, à qui ont longtemps été préférées en France les expressions moins abstraites de différence des sexes, rapports sociaux de sexe, relations entre les hommes et les femmes, masculinféminin. Une historienne utiliserait d'ailleurs plus volontiers gender turn pour signifier ces évolutions internes que pour mesurer l'investissement de la discipline dans l'approche.

8 En histoire, les résistances sont aussi venues des collègues femmes qui s'investissaient dans des recherches considérées comme apportant plus de légitimité professionnelle et craignaient d'être taxées de féministes.

9 Point de comparaison : en 1984,A. Farge pratiquait un comptage dans la prestigieuse revue des Annales esc pour les années 1970 à 1982. Elle dénombrait 4,5\% d'articles concernant les femmes ou des thèmes les mettant au premier plan. II est vrai que l'intérêt des Annales pour les modes de parenté augmentait le pourcentage qui n'était que de 2 à $3 \%$ dans la jeune revue L'Histoire née en 1978 (pour cette revue qui comprend des articles de taille très différente, la surface rédactionnelle a servi d'instrument de mesure). 
La jeunesse des SIC et celle, plus manifeste encore, du questionnement femmes et genre dans cette discipline permettront sans doute d'aller plus vite. Cueillant les fruits des débats les plus récents sur la fluidité des identités de genre ou sur les relations entre sexualisation des questions raciales et racialisation des questions sexuelles, il est possible que les sIC se laissent assez rapidement « gagner par le trouble du genre » et acceptent l'idée que toute communication est par définition genrée (Marie-Joseph Bertini) et que le genre est un défi à la pensée binaire. Je le souhaite vivement mais pense aussi que «la déconstruction des impensés » peut emprunter des voies multiples qui auront leur utilité. Pour ma part et ma discipline, je préfère éviter une définition trop figée et évoquer des usages multiples du concept (sept au moins), qui opèrent des déplacements heuristiques en histoire des femmes et en histoire générale ${ }^{10}$.

Avant de passer à l'examen des politiques du genre en sciences humaines à partir de l'exemple de la discipline historique en France, je tiens à signaler que je partage l'irritation de Marlène Coulomb-Gully devant les réticences exprimées par les collègues et les instances administratives à la féminisation des titres. Mais j'appelle aussi à remplacer l'initiale des bibliographies par toutes les lettres du prénom, le sexe du chercheur étant, me semble-t-il, une information non négligeable...'

\section{Examen des politiques du genre en sciences humaines}

D'emblée, ce programme évoque plus de significations du côté américain et canadien de l'Atlantique que du côté français, où il suscitera sans doute chez les historiens une certaine incompréhension et méfiance. La référence à une science universelle y a longtemps masqué et y masque encore partiellement la question de la place des femmes dans les disciplines et les métiers correspondants, comme celle de la sexuation des savoirs. Une analyse politique, au sens large de définition des savoirs dominants et de répartition des pouvoirs dans les institutions, y apparaît souvent incongrue, au-delà de l'observation des politiques publiques en matière de recherche et d'enseignement.

Dans Écrire l'histoire des femmes et du genre, réédition complétée d'un ouvrage antérieur (Thébaud, 2007), j'ai proposé le récit d'une aventure

\footnotetext{
${ }_{11}^{10}$ Ce point est évoqué plus longuement dans Thébaud (2007).

1 Cette information permet de réfléchir à la place des femmes dans les sciences même s'il est tout aussi important de se demander, avec D. Gardey (Gardey, Löwy, 2000 ; Gardey, 2006), ce que les sciences ont fait et font aux femmes.
} 
intellectuelle de plus de trois décennies ${ }^{12}$. J'entendais ainsi contribuer en France au processus de reconnaissance de ce domaine de recherches, par la transmission d'une expérience historiographique, l'exposé des débats méthodologiques et une mise en perspective internationale. À la fois par choix (préférer la défense et illustration d'une approche novatrice au développement de thèmes jugés polémiques) et par contrainte (absence de travaux significatifs en histoire ou sociologie), j'y ai largement laissé de côté les aspects institutionnels et humains, seulement esquissés par ailleurs (Thébaud, 2004b, 2005b) ${ }^{13}$ : parcours, carrières et engagements de ses chercheuses (la place des hommes y est récente et minoritaire), lieux d'ancrage institutionnel (revues, pôles de développement), politiques académiques et éditoriales.

Derrière Joan Scott (1988a), qui invite à comprendre en quoi la politique définit le genre (place respective et hiérarchisée des hommes et des femmes, conceptions culturelles du masculin et du féminin), et en quoi le genre structure la politique, il s'agira de reprendre sous un autre angle ce savoir accumulé ; en prêtant attention notamment aux définitions de la discipline et à ses contestations, au rôle des actrices (scientifiques femmes), aux moments singuliers où changent les configurations de savoir et de pouvoir, aux résistances multiples et à leurs recompositions. L'approche sera chronologique, évoquant d'abord le poids d'une discipline longtemps masculine, distinguant ensuite un moment féministe où s'impose une féminisation des savoirs et de ses producteurs, avant d'observer le cheminement et les usages d'une histoire du genre. Une quatrième partie précisera les paradoxes du paysage français d'aujourd'hui qui ne signe pas, loin de là, une success story sans failles.

\section{Une discipline masculine, un métier d'hommes}

Pendant des siècles, le silence de l'histoire officielle sur le passé des femmes fut pesant, sauf à évoquer quelques figures exceptionnelles en beauté, en vertu ou en méchanceté, panégyrique des femmes illustres et dénonciation des excès du caractère féminin. Redécouvertes récemment, des femmes de lettres ont tenté d'y répondre, faisant de l'écriture d'une histoire au féminin (biographies ou récits de la condition féminine) un enjeu individuel et collectif pour défendre l'égalité des deux sexes, voire la supériorité du sexe féminin. Dans la lignée des travaux pionniers

\footnotetext{
${ }^{12}$ Plusieurs articles synthétiques lui ont été demandés sur ces thématiques. Citons notamment Thébaud (2004a, 2005a). Cette contribution en reprend certains éléments et en développe d'autres.

${ }^{13}$ Voir aussi à ce sujet l'analyse interdisciplinaire plus ancienne de R.-M. Lagrave (1990).
} 
des historiennes américaines - Natalie Zemon Davis $(1976,1980)$ et Bonnie Smith (1998), Isabelle Ernot (2004, 2007) - a repris l'enquête pour la France, observant le champ historique comme un espace de représentations qui accueille la créativité des historiennes ainsi que leurs résistances à l'idée d'une hiérarchie entre les sexes : entre la fin du XVIII siècle et les années 30 , elle a recensé une cinquantaine « d'historiennes 》 des femmes et retrouvé une centaine d'ouvrages, une bonne part des écrits historiques de femmes étant consacrée à leur sexe. Dans la première moitié du $X \mid X^{e}$ siècle, elles s'y expriment notamment à travers des dictionnaires et recueils biographiques dont la disparition postérieure, au profit d'ouvrages thématiques ou de biographies individuelles, semble signer l'échec des femmes à imposer, après l'instauration du suffrage masculin en 1848, l'idée de l'identité des sexes (Ernot, 2006). Même si les dernières décennies de la période voient surgir les premières thèses de doctorat, la plupart de ces femmes, telles Eugénie Vauvilliers (vers 1775-après 1862), Clarisse Bader ( 1840-1902), ou Arvède Barine (I 8401908) ont été oubliées, faute de légitimité et de transmission.

En effet, tout se fige lorsque l'histoire se constitue en discipline universitaire prestigieuse et au service de la construction nationale républicaine (Carbonell, 1976 ; Noiriel, 1996). Comme l'a très bien montré Bonnie Smith, l'histoirescience et l'histoire-métier se sont construites et définies contre des attitudes considérées comme féminines : investigation méthodique des documents contre l'amateurisme et l'ornement littéraire, primat d'une histoire politique fondée sur la consultation d'archives publiques contre tout discours « légendaire »sur des femmes de pouvoir ou sur le privé, originalité et objectivité contre falsification et copie d'écrits antérieurs. Qu'ils appartinssent à l'école dite méthodique (fin $X \mid X^{e}$ et début du $X X^{e}$ siècle), qui écrivait l'histoire politique et diplomatique des nations et des États, ou aux premières générations des Annales ${ }^{14}$, qui privilégiaient l'économique et le social, les historiens produisirent et enseignèrent alors une histoire au masculin qui se présentait comme l'histoire de l'humanité et ignorait les femmes, considérées par définition comme hors du champ politique et économique. Cette histoire était aussi une histoire asexuée qui oubliait la différence des sexes et le masculin dans l'homme. Même produits conformément aux nouvelles règles du métier, les travaux d'histoire des femmes ne pouvaient avoir de légitimité. Pas plus que les historiennes, « indésirables » comme toutes les femmes dans les universités littéraires jusqu'à la Deuxième guerre mondiale ${ }^{15}$.

\footnotetext{
${ }^{14}$ La revue Annales d'histoire économique et sociale est lancée en janvier 1929 par M. Bloch ${ }_{5}$ et $L$. Febvre qui critiquent vertement la génération précédente.

15 L'adjectif est utilisé par Chr. Charle (1994). Les universités scientifiques ont ouvert leurs portes plus tôt dans des disciplines considérées comme peu théoriques comme la chimie ou les sciences naturelles.
} 
La première diplômée recrutée semble être la byzantinologue Germaine Rouillard, accueillie en 1936 à l'École pratique des hautes études, plus ouverte que la Sorbonne ou les facultés de province.

L'histoire fut ainsi longtemps « un métier d'hommes qui écrivent l'histoire des hommes, présentée comme universelle, tandis que les murs de la Sorbonne se couvrent de fresques féminines » (Duby, Perrot, |99| : |4). Les écoles historiques, les équipes de revues sont des communautés d'hommes qui utilisent le travail gratuit de femmes œuvrant dans l'ombre (très souvent leurs épouses rencontrées sur les bancs des facultés), telle l'école des Annales qualifiée de « confrérie de frères » par Natalie Zemon Davis ( 1992). Lucie Varga, l'assistante au destin tragique ${ }^{16}$ (Schöttler, 199|), et la médiéviste Thérèse Sclafert, dont la thèse remarquée n'eut aucun effet de carrière pour cette enseignante du secondaire, furent les seules femmes à signer un article dans la revue entre sa fondation et la mort de Marc Bloch en 1944. Docteure ès lettres la même année que Thérèse Sclafert, avec une thèse sur Le féminisme dans le socialisme français de 1830 à 1850, Marguerite Thibert sait que l'Université lui est fermée et accepte de partir à Genève comme vacataire au Bureau international du travail ; elle allait y devenir chef du service du travail des femmes et des enfants, puis experte envoyée dans le monde entier pour les questions de formation professionnelle des jeunes filles ${ }^{17}$.

Comme l'écrit Isabelle Ernot (2007 : 176), « l'histoire du terme "historienne" reflète celle de la constitution de la discipline en même temps qu'elle révèle ses caractéristiques sexuées »». Utilisé jusqu’au début du $X I X^{e}$ siècle, il connaît ensuite une longue éclipse, pour réapparaître timidement dans les années 30 , puis plus fréquemment dans les années 50 ; ce que confirme sur le long terme la recherche de mentions dans la première grande revue professionnelle qu'est la Revue historique fondée en 1876. Mais si l'après-Deuxième Guerre est un peu plus ouvert aux femmes chercheuses ${ }^{18}$, il ne l'est qu'à certains profils de femmes et pas encore à l'histoire des femmes. Deux anecdotes concomitantes racontées par les deux pionnières de l'histoire des femmes en France - Michelle Perrot et Yvonne Knibiehler - sont tout à fait significatives à cet égard. En 1949, peu après la sortie du Deuxième Sexe, la première rencontre, pour

\footnotetext{
${ }^{16}$ Jeune juive autrichienne réfugiée en France, L. Varga est l'assistante de L. Febvre qui l'abandonne pour fuir une passion naissante. Ayant perdu appui, relations et travail, elle ${ }_{17}$ doit se cacher pendant la guerre et meurt faute de soins en avril 1941, à 36 ans.

I7 Je consacre actuellement mes recherches au parcours de M. Thibert (1886-1982), 18 intellectuelle, engagée, voyageuse...

${ }^{18}$ On manque de travaux précis sur la féminisation du personnel enseignant des facultés, qui est très faible en lettres en 1946 (3,5\% à Paris, 6,9\% en province).Vingt ans plus tard, le taux est encore inférieur à $20 \%$ (Charle, 1999).
} 
un sujet de diplôme (actuel master), Ernest Labrousse qui représente à la Sorbonne la modernité de l'histoire sociale et quantitative ; ce dernier traduit sa demande de recherche sur le féminisme en exigence « d'un sujet très actuel » et lui propose de travailler sur les coalitions ouvrières de la première moitié du XIXe siècle, histoire ouvrière qui allait être aussi le thème de son doctorat d'État (Perrot, 1987 : 277). Au même moment, l'agrégée Yvonne annonce son mariage à celui qui lui avait choisi un sujet de thèse sur Bernard de Clairvaux ; la réponse fut des vœux de bonheur mais aussi ce « verdict » blessant, « si vous avez des enfants vous serez perdue pour l'histoire »; quinze ans et trois enfants plus tard, assistante à l'université d'Aix-en-Provence, elle dut encore affronter le scepticisme des professeurs hommes qui invoquaient son âge et ses charges de famille, avant d'entamer une thèse d'histoire politique et culturelle sur Adolphe Mignet (Knibiehler, 2007 : 57, 137). Comme le souligne Olivier Dumoulin (1998: 346), l'histoire, plus encore que d'autres disciplines littéraires, est longtemps demeurée « un bastion de résistance masculine 》, avec seulement 3 femmes professeurs sur 120 en 1965 et 10 femmes sur 87 dans le corps des maitres assistants.

\section{Le moment féministe}

Dominante et longtemps incontestable dans l'institution, cette histoire au masculin est devenue critiquable dans un contexte intellectuel, social et politique favorable à l'émergence de nouveaux questionnements et à de multiples contestations, contexte que des historiens ont appelé récemment de façon heuristique « les années 68 », qui englobent une grande partie des décennies 60 et 70 (Dreyfus-Armand et al., 2000). Comme telle, l'histoire des femmes est d'abord fille du féminisme et fille des sciences humaines de son temps. Ces dernières offrent en effet un contexte culturel favorable: parution de travaux pionniers en sociologie du travail féminin (représentée notamment par Andrée Michel, Madeleine Guilbert et Evelyne Sullerot) ; renouvellement de l'anthropologie qui incite les historiens à travailler sur la famille, les relations de parenté et le symbolique ; influence diffuse de Michel Foucault qui défend une démarche antinaturaliste et des objets d'étude originaux (la folie, le pouvoir, les disciplines, les sexualités) ; ouverture de la discipline historique à l'histoire ouvrière et à la « nouvelle histoire », qui propose l'élargissement du champ des recherches aux mentalités, à la démographie, aux marginaux, aux attitudes devant la vie, au quotidien, tous sujets qui rencontrent inévitablement les femmes.

Mais le facteur essentiel d'émergence est, au début des années 70, un facteur politique, doublé d'une réalité sociologique. Animé par des filles du baby boom ayant bénéficié de la première vague de démocratisation de l'enseignement secondaire et supérieur, le mouvement de libération des 
femmes - appelé souvent par les historiens « féminisme de la deuxième vague »-donne une impulsion décisive, en s'interrogeant sur le passé et en cherchant les racines de l'oppression ou de la révolte. II suscite, dans le milieu de la recherche et de l'enseignement, le relais de sympathisantes qui, nées dans la décennie 1920, formées et reconnues en histoire sociale et politique, constituent la génération pionnière en histoire des femmes et proposent les premiers travaux de maîtrise puis de thèse. Deux figures de l'histoire contemporaine ont déjà été citées. Yvonne Knibiehler fonde en 1972 le CEFUP (Centre d'études féminines de l'université de Provence), premier groupe français de recherche sur les femmes (Thébaud, 2004a). Michelle Perrot fait le choix post-soixante-huitard de l'université Paris 7 (Basch et al., 200 I) et organise à l'automne 1973 un cours dont l'intitulé, signe d'une réelle perplexité et d'une volonté de savoir, ouvre une filiation significative : « Les femmes ont-elles une histoire ? », prélude dix ans plus tard du colloque « Une histoire des femmes est-elle possible? » (Perrot, 1984), puis de « Une histoire sans les femmes est-elle possible? » (Sohn, Thélamon, 1998). II faut mentionner aussi - ce qui dessine une géographie durable des pôles français de l'histoire de femmes - Madeleine Rebérioux qui enseigne à l'université Paris 8-Vincennes et Rolande Trempé à l'université Toulouse-Le Mirail, toutes deux développant notamment l'histoire des mouvements sociaux.

Comme l'ont souligné des ouvrages récents (Gubin et al., 2004), le féminisme de la deuxième vague comporte une forte dimension culturelle de critique des savoirs établis. En histoire, les travaux de recherche sont, dès le début, à la fois inscrits dans les développements contemporains de la discipline historique (enracinement de l'histoire moderne des femmes dans l'histoire démographique et de la famille, et de l'histoire contemporaine dans l'histoire sociale et ouvrière ${ }^{19}$ ) et à l'écoute de l'actualité sociale du moment. Ils explorent l'histoire de l'avortement, de la prostitution, des mouvements néo-malthusiens, des féminismes, des relations amoureuses entre femmes, des grèves féminines auX $X I X^{e}$ et $X X^{e}$ siècles, des rapports des féministes avec les mouvements ouvriers. Ils inaugurent ainsi un ambitieux projet de remémoration et de visibilité - redécouvrir le passé des femmes et donner une mémoire à celles d'aujourd'hui -, véritable « histoire au féminin », herstory (et non history) comme disent les Américaines qui peuvent utiliser ce jeu de mots. En engageant un savoir, cette histoire des femmes par les femmes - les hommes sont alors peu nombreux et pas toujours bienvenus -, sur les femmes et pour les femmes fait émerger des sujets occultés : ceux cités précédemment, mais aussi, pour

\footnotetext{
${ }^{19}$ M. Perrot est le meilleur exemple de cette insertion de l'histoire des femmes à ses débuts dans l'histoire sociale, voir Perrot (1998).
} 
des historiennes à la recherche d'une identité féminine, la maternité, le corps, la sexualité, les lieux et la culture de l'entre-femmes, domaines féminins particulièrement explorés parYvonne Knibiehler. Elle comble ainsi les vides d'une histoire officielle et interroge quelques-uns de ses paradigmes, comme les césures chronologiques - quel sens ont tel événement (guerre, révolution) ou telle période (Renaissance, Libération, Trente Glorieuses) du point de vue des femmes ? -, ou la distinction entre une sphère publique digne d'intérêt et une sphère privée indépendante et inaccessible. Elle met aussi au jour la fausse universalité du récit historique ou la trop grande rigidité de l'opposition entre objectivité et subjectivité, soulignant aussi le caractère heuristique de connaissances « situées » (Löwy, 2000). Elle se montre enfin inventive sur les matériaux historiques, apprenant à lire autrement les sources classiques de l'histoire et à rechercher des sources féminines, journaux intimes, correspondances ou témoignages oraux.

On peut dater ces années «féministes 》 de 1972 (création du CEFUP) à 1985 où disparaît Pénélope, pour l'histoire des femmes, après 13 numéros semestriels. Elles constituent une formidable phase d'accumulation des connaissances dans les domaines les plus divers pour lesquels s'engage une génération jeune et motivée, parfois cosmopolite comme à l'université Paris 7 où Michelle Perrot encadre, dans les années 80, les recherches de Grecques, Italiennes Suisses et Brésiliennes. Conquérantes, I'histoire des femmes et ses chercheuses n'en rencontrent pas moins de nombreuses difficultés. Tout d'abord, les rapports entre militantisme et recherche, entre l'institution et le hors institution sont souvent tendus et divers, comme en témoignent les appellations fort variées des groupes de recherche nés dans les années 70 (groupe « d'études féministes 》, 《d'études des femmes 》, « d'études féminines ») ou les débats du colloque de Toulouse «Femmes, féminisme et recherches 》, grand colloque interdisciplinaire qui rassemble, en décembre 1982, 750 participant-e-s et 140 communications. Les historiennes y apparaissent plutôt légitimistes, exprimant la crainte des effets négatifs de l'entresoi et le souhait de l'intégration des « études femmes » aux territoires de l'histoire. Les universitaires les mieux intégrées craignent avant tout l'isolement (le « ghetto intellectuel », selon le terme maintes fois répété) et ne revendiquent pas la création de women's studies à l'américaine ni de postes fléchés en histoire des femmes. Elles sont aussi retenues par les plus militantes, parfois hors institution, qui gardent la méfiance du féminisme français envers l'État et toute tentative de récupération. Ces tensions - encore mal documentées aujourd'hui - inaugurent un mauvais départ en matière d'institutionnalisation et sont sans doute à l'origine d'une occasion manquée pendant les cinq ans du ministère des Droits de la Femme (1981-1986) confié à Yvette Roudy après l'alternance politique de 1981. Les quelques acquis sont en grande partie éphémères 
et plus favorables à la recherche qu'à la transmission : financement par le CNRS (Centre national de la recherche scientifique) d'une soixantaine de projets entre 1984 et 1989, création de trois postes d'études féministes dont un seul en histoire à l'université de Toulouse-Le Mirail20.

Utilisé couramment dans de nombreux pays d'Europe et d'Amérique, le terme « histoire féministe » est d'ailleurs peu usité en France (sauf de façon péjorative) où l'on préfère souligner que, si l'historienne ou l'historien de l'histoire des femmes est souvent féministe, son discours est aussi « scientifique » qu'un autre. C'est que, deuxième difficulté, les résistances rencontrées sont grandes. Les origines féministes de l'histoire des femmes et le développement d'une « histoire au féminin » suscitent alors la méfiance de la discipline historique, qui dénonce subjectivité, idéologie et militantisme, ainsi que de la profession des historiens qui, très masculine dans ses échelons supérieurs et forte du prestige national de l'histoire, dispose du pouvoir de recrutement. Dans « un marché universitaire » marqué (fin des années 70 et une grande partie des années 80) par la contraction des postes vacants après les recrutements massifs de l'après-1968, les jeunes spécialistes d'histoire des femmes ont du mal à convaincre les instances de recrutement, d'autant que change alors le modèle de thèse à la française défendue par certains ${ }^{21}$. De même, sauf exception comme Syros qui ouvre en 1978 la collection « Mémoire des femmes » et réédite des textes de féministes, les éditeurs découvrent tardivement le domaine ${ }^{22}$. L'histoire des femmes reste aussi invisible pour de nombreuses revues universitaires ${ }^{23}$, à l'exception du Mouvement social, et les premiers travaux (mémoires de maîtrise et thèses) restent quasiment inconnus des historiens classiques.

Espace de réflexion, de sociabilité et de transmission, Pénélope, pour l'histoire des femmes illustre ce moment féministe. Fondée par le Groupe de recherche pour l'histoire et l'anthropologie des femmes (composé

${ }^{20}$ M.-Fr. Brive (prématurément décédée en 1993) en est la titulaire. Les deux autres sont accordées à A. Junter-Loiseau (droit, Rennes 2) et Cl. Zaidman (sociologie, Paris 7). L'auteure de ces pages, qui a déjà esquissé l'histoire des années Roudy (Thébaud, 200I) et participe aujourd'hui à des comités sur l'égalité professionnelle dans l'enseignement supérieur et la recherche, a le projet de creuser cette question.

${ }^{21}$ La thèse de 3 e cycle puis la thèse d'Université (sur le modèle du Ph. D.) sont dévalorisées aux yeux de recruteurs dotés de thèses d'État. La réforme datant de 1984, les jeunes spécialistes d'histoire des femmes ne sont pas docteur-e-s d'État.

22 Ils créent alors des collections devant le bon accueil du public, plus réceptif que l'establishment universitaire. Parmi les collections ouvertes dans les années 80, citons « La femme au temps de... » chez Stock.

${ }^{23}$ Dans la célèbre revue des Annales ESC, A. Farge a recensé, pour la période 1970- 1982, 75 | articles, dont 139 signés par des femmes (taux de 18,5\% correspondant à leur place dans la profession) et seulement 34 en histoire des femmes, soit 4,5\% (moins encore si on ne compte pas les articles portant sur la problématique des structures de parenté). 
de chercheuses du Centre de recherche historique de la Maison des sciences de l'homme et du Groupe d'études féministes de l'université Paris 7), la revue accompagne, après un numéro zéro commun avec le CEFUP et le CLEF lyonnais, les recherches d'histoire au féminin et les premières soutenances de thèses. Sans éditeur, imprimée par l'atelier de reprographie de l'université Paris 7 puis par une imprimerie de femmes, elle est à la recherche d'un difficile équilibre entre militantisme et recherche (faire de l'histoire et aider le mouvement des femmes). Comme l'indique l'éditorial du premier numéro, Pénélope ne veut pas être « une revue académique, exhaustive et fignolée », mais plutôt de « modestes cahiers », qui réunissent l'information de façon thématique, la font circuler et stimulent « la réflexion sur cette dimension de l'Histoire qu'est l'histoire des femmes et sur cette dimension qu'est pour les femmes leur temps collectif ». Elle est née aussi, ce qui est souvent oublié, de l'échec d'une tentative de revue internationale d'histoire des femmes proposée par un éditeur américain : la différence de moyens universitaires de part et d'autre de l'Atlantique, l'usage exclusif de l'anglais imposé par l'éditeur, l'ambition d'une diffusion européenne ont fait échouer le projet mûri lors du premier congrès international d'histoire des femmes auquel participent des Françaises en novembre 1977 (Dauphin, 200 I).

Gage de légitimité et d'échanges fructueux, la dimension internationale du domaine de recherche et la circulation des personnes et des idées s'accompagnent, dès le début, à la fois d'une dynamique commune et de décalages, voire d'incompréhensions, renforcés par des traductions tardives et sélectives en France, plus rapides mais plus sélectives encore aux États-Unis. Un autre exemple, qu'il serait d'ailleurs intéressant de mieux documenter en interrogeant les protagonistes du projet, est significatif à cet égard: organisées à l'initiative de Carrol Smith-Rosenberg et avec l'aide de la Fondation Rockefeller, des rencontres en France et aux États-Unis entre des chercheuses françaises, américaines, italiennes, anglaises, allemandes et néerlandaises (1979-1982) aboutissent à la publication de deux livres collectifs, à la fois proches et différents : paru en 1984, Stratégies des femmes (Pasquier et al.) problématisent l'identité sexuelle et insiste sur les réponses féminines et féministes dans des contextes donnés ; publié deux ans plus tard, Women in Culture and Politics: A Century of Change (Friedlander et al., 1986) ajoute quelques contributions et structure l'ouvrage autour des deux approches culturelle et politique - qui se confrontent alors depuis une dizaine d'années aux États-Unis. Américaine enseignant en France, Rebecca Rogers confirme ces difficultés dans son analyse récente de plusieurs décennies d'appropriations réciproques (2004). II en est de même pour ce qu'on appelle aujourd'hui l'histoire du genre, au sujet de laquelle furent et sont encore grandes les divergences théoriques entre ÉtatsUnis et France. 


\section{Vers une histoire du genre : circulations internationales et spécificités françaises}

Le glissement d'une histoire au féminin, qui stimule encore aujourd'hui des recherches neuves sur des aspects mal connus, à une gender history tardivement traduite en France par « histoire du genre », résulte à la fois de critiques internes au champ de recherche et d'un phénomène de transfert et accommodement de notions venues d'outre-Atlantique et d'autres disciplines (psychologie et sociologie notamment). En effet, l'histoire des femmes a toujours réfléchi à la manière d'en écrire les résultats, développant un débat interne ponctué de colloques et d'articles fondateurs, recherchant les échanges interdisciplinaires et internationaux, suscitant un dialogue externe avec les historiens d'autres spécialités.

Les années 80 formulent deux critiques et posent deux questions aux approches précédentes. Première critique : l'histoire proposée ne simplifie-t-elle pas la réalité au profit d'une histoire du malheur et de la révolte des femmes qui invoque la domination masculine sans en expliciter le fonctionnement et au détriment de la recherche de ce que Cécile Dauphin et Arlette Farge (200I : 8) allaient appeler plus tard «le nuancier infini de la rencontre entre hommes et femmes »? De même, posée notamment par l'ouvrage de Bonnie Smith sur les bourgeoises du Nord (1981, traduction 1989), la thématique stimulante d'une « culture féminine », qui permet d'identifier des lieux, conduites ou objets féminins, des pouvoirs possédés par les femmes, des espaces de liberté et de relations entre femmes, comporte le risque de réassurer le mythe d'une nature féminine et d'occulter la domination masculine par le thème de la complémentarité (Dauphin et al., 1986). Plus largement, quel sens y a-t-il à faire des femmes un objet autonome d'histoire et celles-ci constituentelles un groupe social homogène ? Ne faut-il pas mieux, en mettant le rapport entre les sexes et la construction du masculin et du féminin au cœur de l'interrogation historique, incorporer l'histoire des femmes à I'histoire générale et transformer celle-ci?

Si le concept et le terme de gender - dont il ne sera pas fait ici l'histoire restent longtemps peu connus en France, pas du tout utilisés avant la traduction en français en 1988 de l'article de Joan Scott « Gender : A Useful Category of Historical Analysis $\gg^{24}$ et fortement discutés ensuite, l'histoire des femmes a su en intégrer une partie des apports méthodologiques et conceptuels, proposer une histoire relationnelle du rapport entre les hommes et les femmes et une lecture sexuée

${ }^{24}$ « Gender : A Useful Category of Historical Analysis » de J. Scott est paru deux ans plus tôt dans The American Historical Review. 
des événements et phénomènes historiques, comme en témoignent les cinq volumes de la collection Histoire des femmes en Occident (Duby, Perrot, 1991-1992). Première tentative de synthèse des travaux et des questionnements des vingt années précédentes et essai d'histoire comparative, elle représente au début des années 90 le gender à la française, avec des caractéristiques soulignées par les commentaires étrangers : choix de la longue durée et des coupures chronologiques des grandes collections françaises, mixité des auteurs (I I hommes sur 76) et surtout approches plurielles sans théorie préliminaire autre qu'un minimum de convictions partagées et exposées en introduction : faire l'histoire des relations entre les sexes « à tous les niveaux de la représentation, des savoirs, des pouvoirs et des pratiques quotidiennes ». Les débats du colloque de la Sorbonne (Duby, Perrot, 1993), qui en proposent des lectures critiques contradictoires, offrent une version très feutrée des oppositions contemporaines en terre anglophone, entre histoire sociale et histoire culturelle, entre women's history et gender history, entre gender history avec « conception sciences sociales du gender $\gg$ et gender history post-structuraliste ${ }^{25}$. Ils montrent que l'approche française reste empirique et pluraliste, méfiante envers le linguistic turn et ses débats théoriques mais sachant intégrer une attention au langage dans une approche d'histoire sociale, sensible aux différences dans la différence et à l'articulation des différentes catégories d'analyse (sexe, âge, classe, religion, nationalité, etc.). Au colloque ou hors colloque, quelques historiens ou historiennes françaises dénoncent cependant la place excessive accordée aux discours et aux représentations, l'oubli des parcours singuliers ou des différences entre femmes, ou bien la complaisance envers la description des pratiques culturelles et des actes de la vie quotidienne ; ainsi Christine Fauré (1997) qui entreprend alors une encyclopédie politique et historique des femmes.

L'histoire de ce projet éditorial, qui traduit le statut longtemps marginal du domaine de recherche, mérite d'être racontée. L'initiative n'en est pas française mais italienne : sensible à l'air du temps et heureux traducteur de L'Histoire de la vie privée (Ariès, Duby, 1986-1987), l'éditeur Laterza s'adresse à Georges Duby qui contacte Michelle Perrot pour constituer une équipe de direction française. La version française est cependant refusée par Le Seuil, prestigieuse maison coutumière des « grandes collections » en quatre ou cinq volumes. Inattendu, le succès de l'entreprise, tant en France ${ }^{26}$ (20 000 exemplaires rapidement écoulés) qu'à l'étranger

\footnotetext{
${ }_{26}^{25}$ Pour plus d'informations, voir Thébaud (2007).

${ }^{26}$ C'est finalement Plon qui accepta le projet, grâce au soutien de L. Adler qui y travaillait alors.
} 
où une dizaine de traductions ${ }^{27}$ suscite parfois des entreprises parallèles, contribue indéniablement à la reconnaissance intellectuelle du champ de recherche : il devient alors difficile de soutenir que les femmes n'ont pas d'histoire, qu'il est impossible de l'écrire (faute de sources), ou que le public lecteur - pour qui l'égalité entre hommes et femmes est une question vive des sociétés contemporaines - ne s'y intéresse pas. Ce contexte facilite la création en 1995 de la deuxième revue française CLIO, Histoire, femmes et sociétés, qui, à l'inverse des Cahiers du Mage (marché du travail et genre), choisit, après mûre réflexion et par crainte de ne pas être compris, de ne pas introduire le mot « genre » dans son sous-titre. Il permet aussi à quelques jeunes diplômées en histoire des femmes parfois dotées d'allocations de recherche pendant leurs études - d'obtenir un poste au milieu des années 90 à la faveur de recrutements consécutifs à la croissance du nombre d'étudiants.

Projet personne ${ }^{28}$ devenu réalisation collective entre 1993 et 1995 , CLIO, Histoire, femmes et sociétés n'est pas une revue Pénélope bis et son développement s'inscrit dans une autre configuration de savoir et de pouvoir. Certes, la revue appartient d'abord à son Comité de rédaction, aucune institution - laboratoire ou centre de recherche - n'existant en histoire des femmes en France. Mais, dotée d'un véritable éditeur et subventionnée, elle s'inscrit d'emblée dans le champ académique, soucieuse de la qualité scientifique de son contenu et du respect des normes du métier. Libérée de la crainte, autrefois affirmée, de faire de l'histoire des femmes un « ghetto », animée de la volonté d'obtenir une reconnaissance intellectuelle et institutionnelle de l'histoire des femmes et du genre, elle affirme, par son titre et son contenu, son appartenance à la communauté des historiens, joue la carte de la visibilité intellectuelle et recherche une confrontation mutuelle féconde avec à la discipline historique. Attentive à faire dialoguer les approches (histoire des femmes et histoire du genre, histoire sociale et histoire culturelle, histoire politique et anthropologie historique) et à faire connaitre les travaux étrangers, elle offre un lieu d'expression et de débat qui s'impose peu à peu dans le paysage intellectuel des revues d'histoire. Elle prépare aujourd'hui, toujours sans secrétaire de rédaction, son $28^{e}$ numéro et offre en ligne les dix premières années de sa collection sur le site très consulté de revues.org. Situation paradoxale à l'image du paysage français actuel.

\footnotetext{
27 Traductions simples, ou avec ajout de préfaces et/ou de chapitres complémentaires.

${ }^{28}$ Directrice éditoriale du $5^{e}$ tome de l'Histoire des femmes en Occident (volume sur le $X X^{e}$ siècle), alors lectrice occasionnelle des premières revues anglophones d'histoire des femmes ou du genre (Gender \& History, Women's History Review) et de jeunes revues francophones interdisciplinaires (la québécoise Recherches féministes, la belge Sextant), au courant du projet espagnol d'Arenal, revista de historia de las mujeres, j'ai pris l'initiative de créer en France un périodique qui allait devenir Clio hfs.
} 


\section{Les paradoxes du paysage actuel}

Spécialiste d'histoire politique, Michèle Riot-Sarcey (2000) a souvent souligné les réticences de l'historiographie française à l'égard du genre, particulièrement envers l'approche de Joan Scott, qui, politique - «le genre est une façon première de signifier des rapports de pouvoir » écrit-elle - et culturelle, invite à mettre l'accent moins sur les parties (les hommes et les femmes) que sur le principe de partition et ses significations. S'il est vrai que la critique des historiens sociaux - oubli de la réalité socio-politique des femmes, échappée hors des territoires de la discipline historique - a du poids en France, d'autres collègues soulignent au contraire l'intérêt de l'historicisation des discours, la complémentarité des approches sociales et discursives et les enjeux de réalité des luttes de représentation (Chartier, 1998 ; Ernot, 2004). Plus généralement, les acceptions et usages du genre sont divers et objet de débats, interpellant à la fois l'histoire des femmes et l'histoire générale, faisant de ce champ de recherche un lieu vivant, dynamique, ouvert sur l'interdisciplinarité et les échanges avec l'étranger. Si sa légitimité institutionnelle est mal assise (voir plus loin), si sa reconnaissance intellectuelle est encore parfois contestée, il constitue, pour reprendre les mots d'Alain Corbin, un indéniable « laboratoire de novations $»^{29}$.

Inscrit dans un partage entre nature et culture et dans une perspective constructiviste, le genre dit d'abord que la condition et l'identité des femmes ne se comprend que dans la relation aux hommes et qu'elles sont le résultat d'une construction sociale et culturelle dans un contexte donné : «la femme » n'existe pas - sauf dans l'imaginaire des hommes ou ... des femmes - et la tâche de l'histoire est de comprendre l'évolution des systèmes de genre, ensembles de rôles sociaux sexués et systèmes de représentation définissant le masculin et le féminin. Le genre implique aussi qu'il n'y pas de sexe que féminin et rend visibles les hommes comme individus sexués. À l'encontre d'une dissymétrie de l'histoire des femmes très tôt pointée par Alain Corbin (1984) - mais on peut dire aussi que sans I'histoire des femmes, celle des hommes n'était pas pensable -, il a ainsi suscité depuis une dizaine d'années en France l'émergence d'une histoire des hommes et des masculinités, qui examine la construction de la virilité et observe les positions dominantes comme les souffrances des hommes (Roynette, 2000 ; Revenin, 2007). Cela débouche aujourd'hui sur le projet, complexe mais stimulant, d'une histoire des identités sociales et sexuées, à travers l'historicisation des identités, l'analyse des formes de confrontation des individus aux modèles identitaires dominants, ou l'observation des

${ }^{29}$ Qualification utilisée par A. Corbin dans la préface rédigée en 1998 pour la première édition de l'ouvrage de Fr.Thébaud (2007 [1998]). 
crises d'identité dans des moments de rupture (Capdevila et al., 2003). Confronté à d'autres catégories d'analyse comme la classe sociale mais aussi l'appartenance nationale ou religieuse, le groupe d'âge, la « race » ou l'orientation sexuelle, le genre invite enfin à réfléchir aux différences entres femmes. Stimulés par des travaux étrangers et des débats de société, les historiens et historiennes français commencent d'ailleurs à ne plus être aveugles à la différence de « race » et à envisager dans toute sa complexité une histoire nationale marquée par l'immigration et la colonisation (colloques récents et thèses en cours).

Concernant tout autant l'histoire générale, le genre propose une relecture sexuée des événements et phénomènes historiques qui contribue à leur explication. S'interroger sur « le genre de... » la nation, la citoyenneté, la protection sociale, la colonisation ou bien encore du travail, du militantisme, des sciences ou des migrations ne concerne pas seulement l'observation de la place respective des hommes et des femmes mais aussi l'analyse des enjeux de signification de la division entre masculin et féminin et des modes de construction des rapports sociaux hiérarchiques. Émergeant du questionnement queer sur la fluidité des identités - son ouvrage pionnier vient seulement d'être traduit en français (Butler, 2005) -, le dernier usage tire le fil d'une construction culturelle et sociale qui implique de possibles distorsions entre sexe anatomique, genre social et sexualités. La prégnance dans la culture occidentale d'une norme de correspondance sexe-genre (et de l'idée du caractère explicatif du sexe biologique) a conduit à négliger, en histoire comme dans maintes disciplines, les questions du travestissement qui ont donné quelques travaux récents (Steinberg, 200 I), des homosexualités, de l'androgynie, des pratiques transgenre, des variations intragenre. Ce fil conduit aussi c'est l'objet de plusieurs séminaires aujourd'hui - à écrire l'histoire de la dissociation entre sexe et genre, « une histoire entre nature et culture » comme le titre en 2003 un numéro des Cahiers du Genre.

Comme l'indique le titre de cette revue interdisciplinaire, modifié en 1999, le terme « genre », traduction de l'anglais gender, s'impose en France dans les sciences humaines à l'aube des années 2000. Plus encore que leurs collègues d'autres disciplines, les historiens et historiennes ont longtemps préféré les expressions moins abstraites de différence des sexes, relations entre les hommes et les femmes, masculin-féminin ou rapports sociaux de sexe en usage chez les sociologues marxisants. Là encore, les années 2000 semblent changer la donne. Ainsi Mnémosyne, fondée en 2000, est l'Association pour le développement de l'histoire des femmes et du genre, section française de la Fédération internationale pour la recherche en histoire des femmes qui a vu le jour en 1987. Le laboratoire d'histoire de l'université Rennes 2 réunit en septembre 2002 le premier colloque d'historiens qui affiche le terme sans ambiguïtés: 
« Le genre face aux mutations du Moyen Âge à nos jours » (Capdevila et al., 2003). La même année, tandis que Le Mouvement Social préfère titrer « Féminin et Masculin », Vingtième siècle. Revue d'histoire publie un numéro spécial « Histoire des femmes, histoire des genres », tendance amplement confirmée depuis lors. Cependant, c'est au moment où l'emploi du terme se généralise et suscite un utile travail d'explicitation du concept qu'est rendu l'avis négatif de la Commission générale de terminologie et de néologie placée auprès du Premier ministre. Publié au Journal Officiel du 22 juillet 2005, l'avis déconseille l'emploi de « genre » pour traduire « gender » et les définitions données de ce terme, considéré comme un néologisme et un anglicisme, sont très en retrait de la richesse de ses usages et de ses potentialités. Défense de la langue française ? Collusion entre langue et idéologie nationale ? Antiaméricanisme? Résistance aux études de genre ? Tout au moins, situation paradoxale ${ }^{30}$.

Acquise depuis les années 80 auprès du public lecteur, la reconnaissance intellectuelle de l'histoire des femmes et du genre a indéniablement progressé ces dernières années dans la communauté des historiens: ouverture des revues généralistes d'histoire qui publient plus régulièrement articles et comptes rendus ou organisent occasionnellement un numéro spécial ; insertion dans les manuels d'historiographie récents de chapitres sur l'histoire des femmes; présence dans certains dictionnaires critiques de la dernière génération d'entrées sur les femmes, les rapports de sexe ou le féminisme; publication des quatre premiers manuels universitaires qui atteste d'un développement, réel ou attendu, dans l'enseignement. Cette reconnaissance intellectuelle n'est cependant pas totale et n'est pas suivie d'une reconnaissance institutionnelle qui permet à un domaine de recherche d'exprimer toutes ses potentialités, de se confronter avec d'autres dans un dialogue égal et constructif, de pénétrer les formes habituelles du savoir. Autre paradoxe français : depuis trente à quarante ans, le monde des historiens français a relativement bien absorbé le choc de la féminisation, le sexe du chercheur devenant un facteur de moins en moins discriminant au recrutement (Thébaud, 2003), mais les résistances sont fortes à l'inclusion du domaine de recherches (et de ses promotrices) dans les institutions de recherche et d'enseignement, et donc à la production et plus encore à la transmission d'une culture mixte. Ces résistances suscitent un militantisme académique qui est une des facettes actuelles du lien entre recherche et féminisme : celui-ci continue d'irriguer le questionnement, de susciter de nouvelles thématiques de

\footnotetext{
${ }^{30}$ Sur ce point, voir « Le "genre" interdit », Travail, genre et sociétés, 16, nov. 2006, rubrique «Controverse » (contributions de Th. Locoh et M. Méron, Cl. Baudino, H. Dagenais, E. Hofmann, M. Bozon).
} 
recherche ${ }^{31}$, d'en utiliser les résultats, mais la discipline revendique aujourd'hui l'autonomie relative de tout champ intellectuel par rapport au mouvement social.

\section{Conclusion}

Le paysage actuel est donc très contrasté. Certes, la très grande majorité des étudiants des 87 universités françaises, futurs enseignants pour certains d'entre eux, effectuent leur scolarité sans entendre parler de cette approche de l'histoire et l'ensemble des établissements ne dispose que de quatre postes spécifiques ${ }^{32}$. Mais depuis une dizaine d'années, une diffusion par capillarité diminue le poids relatif des quelques pôles traditionnels et diversifie les lieux universitaires de l'histoire des femmes et du genre, comme les formes de son implantation : au gré de nominations souvent provinciales, apparaissent des cours optionnels, des séminaires, des colloques, des axes de recherche dans de petits établissements ou des universités sans tradition dans ce domaine (une dizaine environ). Après l'adoption de conventions pour l'égalité se met lentement en place, dans les instituts de formation des maîtres, une initiation au genre et à l'égalité des sexes. Moins nombreuses mais toujours inventives, les recherches doctorales en histoire contemporaine - période de l'histoire privilégiée dans cette contribution continuent sous la houlette d'une dizaine d'universitaires habilitées, tandis que de jeunes associations tentent de pallier ce déficit d'institutionnalisation et jouent la carte du dialogue et de la visibilité intellectuelle ${ }^{33}$. Élément d'un possible développement, une charte pour l'égalité, qui croise la réflexion sur la place des femmes dans les carrières scientifiques et l'approche de genre dans les disciplines, vient d'être adoptée par l'université Lyon I et sera prochainement soumise aux autres établissements. L'histoire continue...

\footnotetext{
31 Par exemple, la mobilisation paritaire des années 90 a suscité de nombreux travaux sur la place des femmes dans les espaces publics, et l'affirmation publique des mouvements homosexuels des recherches sur le lesbianisme. De même, la pensée queer interroge la distinction sexe/genre...

322 postes de maîtres de conférences : universités Paris 8 et Paris $7 ; 2$ postes de professeur: Paris 8 et IUFM de Lyon. Le fléchage du poste de Toulouse (obtenu en 1984) a disparu, mais cette université propose aujourd'hui l'offre de cours la plus complète et dispose d'un groupe de recherche interdisciplinaire dynamique dans lequel l'histoire est partie prenante ${ }_{33}$ (le groupe Simone).

33 Association pour le développement de l'histoire des femmes et du genre-Mnemosyne ; SIEFAR (Société internationale d'études des femmes de l'Ancien régime ; Archives du féminisme ; Efigies (Association de jeunes chercheurs en études féministes, genre et sexualités).
} 


\section{Références}

Ariès Ph., Duby G., dirs, 1986-1987, Histoire de la vie privée, Paris, Éd. Le Seuil (5 volumes).

Bard Ch., 2003, « Jalons pour une histoire des études féministes en France (19702002) », Nouvelles questions féministes, vol. 22, I, pp. I4-30.

Basch Fr., Bruit L., Dental M., Picq Fr., Schmitt-Pantel P., Zaidman Cl., dirs, 200I, Vingt-cing ans d'études féministes. L'expérience Jussieu, Paris, CREDEF, Publications de l'Université Paris 7.

Bereni L., Chauvin S., Jaunait A., Revillard A., 2008, Introduction aux Gender Studies. Manuel des études sur le genre, Bruxelles, De Boeck.

Butler J., 1990, Trouble dans le genre. Pour un féminisme de la subversion, trad. de l'américain par C. Kraus, Paris, Éd. La Découverte, 2005.

Capdevila L., Rouquet Fr. Rouquet, Vigili F., Voldman D., 2003, Hommes et femmes dans la France en guerre (19/4-1945), Paris, Payot.

Capdevila L., Cassagnes S., Cocaud M., Godineau D., Rouquet Fr., Sainclivier J., dirs, 2003, Le genre face aux mutations. Masculin et féminin, du Moyen Âge à nos jours, Rennes, Presses universitaires de Rennes.

Carbonell, Ch.-O., 1976, Histoire et historiens. Une mutation idéologique des historiens français, 1865-1885, Toulouse, Privat.

Cavallaro D., 2003, French Feminist Theory, London \& New York, Continuum.

Charle Chr., 1994, La République des universitaires 1870-1940, Paris, Éd. Le Seuil.

— 1999, « Les femmes dans l'enseignement supérieur. Dynamiques et freins d'une présence 1946-1992 », pp. 84- 105, in : Duclert V., Fabre R., Fridenson P., dirs, Avenirs et avant-gardes en France, $X I X^{e}-X X^{e}$ siècles, Paris, Éd. La Découverte.

Chartier R., 1998, Au bord de la falaise. L'histoire entre certitudes et inquiétude, Paris, A. Michel.

Collin Fr., 1988, « Sexes et savoir », Les Cahiers du GRIF, « Le genre de l'histoire », 37-38, pp. 5-7.

Corbin A., 1984, « Le «Sexe en deuil» et l'histoire des femmes au XIXe siècle », pp. I42-I54, in : Perrot M., dir., Une histoire des femmes est-elle possible? ? Marseille, Éd. Rivages.

Dauphin C., 200I, «Pénélope : une expérience militante dans le monde académique », pp. 6I-68, in : Basch Fr., Bruit L., Dental M., Picq Fr., Schmitt-Pantel P., Zaidman Cl., dirs, Vingt-cinq ans d'études féministes. L'expérience Jussieu, Paris, CREDEF, Publications de l'Université Paris 7.

Dauphin C., Farge A., dirs, 200I, Séduction et sociétés. Approches historiques, Paris, Éd. Le Seuil.

Dauphin C., Farge A., Fraisse G., Klapisch-Zuber Ch., Lagrave R.-M., Perrot M., Pézerat P., Ripa Y., Schmitt-Pantel P.,Voldman D., 1986, « Culture et pouvoir des femmes : essai d'historiographie », Annales. Histoire, Sciences sociales, mars-avr., 2, pp. 27I-293. 
Davis Zemon N., 1976, «Women's History in Transition : the European Case », Feminist Studies, vol. 3, 3-4, pp. 83- 103.

- 1980, « Gender and genre: Women as Historical Writers, | 400- | 820 », pp. 153-182, in : Labalme P. A., Beyond their Sex : Learned Women of the European Past, New York up.

— 1992, «Women and the world of the Annales », History Workshop, 33, pp. 121-137.

Dreyfus-Armand G., Frank R., Lévy M.-Fr., Zancarini-Fournel M., dirs, 2000, Les Années 68. Le temps de la contestation, Bruxelles, Éd. Complexe.

Duby G., Perrot M., 1991, « Écrire l'histoire des femmes », introduction à Histoire des femmes en Occident, vol. I, L'Antiquité, Paris, Plon.

- dirs, 1991-1992, Histoire des femmes en Occident, Paris, Plon (5 volumes).

Duchêne A., Moïse Cl., 2009, Langage, genre et sexualité, Montréal, Éd. Nota Bene.

Dumoulin O., 1998, « Archives au féminin, histoire au masculin. Les historiennes professionnelles en France, 1920- 1965 », pp. 343-356, in : Sohn A.-M,Thélamon Fr., L'Histoire sans les femmes est-elle possible? Paris, Perrin.

Ernot I., 2004, « Historiennes et enjeux de l'écriture de l'histoire des femmes ( $179 \mid$ - 1948) », Thèse de doctorat, Université Paris 7 (à paraître aux Presses universitaires du Mirail).

- 2006, « Masculin/féminin dans les dictionnaires et recueils de biographies féminines (début XIXe siècle-années 1860) », pp. 67-84, in : Pellegrin N., Histoires d'historiennes, Saint-Étienne, Publications de l'université de Saint-Étienne.

— 2007, « L'histoire des femmes et ses premières historiennes », Revue d'histoire des sciences humaines, 16, pp. 165-194.

Farge A., 1984, « Pratiques et effets de l'histoire des femmes », pp. 18-35, in : Perrot M., dir., Une histoire des femmes est-elle possible ?, Marseille, Éd. Rivages.

Fauré Ch., dir., 1997, Encyclopédie historique et politique des femmes, Paris, Presses universitaires de Nancy.

Friedlander J., Wiesen Cook Bl., Kessler-Harris A., Smith-Rosenberg C., dirs, 1986, Women in Culture and Politics. A Century of Change, Bloomington, Indiana University Press.

Gardey D., 2006, « Les sciences et la construction des identités sexuées. À propos des corps féminins et de l'histoire des sciences et des techniques », Annales. Histoire, Sciences sociales, 3, mai-juin, pp. 649-673.

Gardey D., Löwy l., dirs, 2000, L'invention du naturel. Les sciences et la fabrication du féminin et du masculin, Paris, Éd. des Archives contemporaines.

Gubin E., Jacques C., Rochefort Fl., Studer Br., Thébaud Fr., Zancarini-Fournel M., dirs, 2004, Le siècle des féminismes, Paris, Éd. de l'Atelier.

Knibiehler Y., 2007, Qui va garder les enfants? Mémoires d'une féministe iconoclaste, Paris, Calmann-Lévy.

Lagrave R.-M., 1990, « Recherches féministes ou recherches sur les femmes 》, Actes de la recherche en sciences sociales, 83, juin, pp. 27-39. 
Löwy l., 2000, « Universalité de la science et connaissances situées 》, pp. I 36- I50, in : Gardey D., Löwy l., dirs, L'invention du naturel. Les sciences et la fabrication du féminin et du masculin, Paris, Éd. des Archives contemporaines.

Noiriel G., 1996, Sur la « crise » de l'histoire, Paris, Belin.

Nouvelles questions féministes, 1996, « France, Amérique : regards croisés sur le féminisme », vol. 17, I.

Pasquier M.-Cl., Marini M., Ducrocq Fr., Fraisse G., Sohn A.-M., dirs, 1984, Stratégies des femmes, Paris, Tierce.

Perrot M., dir., 1984, Une histoire des femmes est-elle possible?, Marseille, Éd. Rivages.

— 1987, « L'air du temps », pp. 24 I-292, in : Nora P., dir., Essais d'ego-histoire, Paris, Gallimard.

— 1998, Les Femmes ou les silences de l'histoire, Paris, Flammarion.

Revenin R., dir., 2007, Hommes et masculinités de 1789 à nos jours. Contributions à l'histoire du genre et de la sexualité en France, Paris, Éd. Autrement.

Roynette O., 2000, Bons pour le service. L'expérience de la caserne en France à la fin du XIXe siècle, Paris, Belin.

Riot-Sarcey M., 2000, « L'historiographie française et le concept de "genre" », Revue d'histoire moderne et contemporaine, vol. 47, 4, pp. 805-8I4.

Rogers R., 2004, « Rencontres, appropriations, résistances : les étapes d'un dialogue franco-américain en histoire des femmes et du genre », Revue d'Histoire des sciences humaines, II, pp. 101-126.

Schöttler P., 1991, Lucie Varga, les autorités invisibles. Une historienne autrichienne aux Annales dans les années trente, Paris, Éd. Le Cerf.

Scott J.W., 1988a, Gender and the Politics of History, Columbia University Press.

— 1988b, « Genre : une catégorie utile d'analyse historique », Les Cahiers du GRIF, 37-38, pp. I25- 153.

Smith B., 1981, Ladies of the Leisure Class. The Bourgeoises of Northern France in the Nineteenth Century, Princeton, Princeton University Press, 1989.

- 1998, The Gender of History. Men, Women and Historical Practice, Cambridge, Harvard University Press.

Sohn A.-M., Thélamon Fr., dirs, 1998, L'Histoire sans les femmes est-elle possible?. Paris, Perrin.

Steinberg S., 200 I, La confusion des sexes. Le travestissement de la Renaissance à la Révolution, Paris, Fayard.

Thébaud Fr., 200I, «Promouvoir les droits des femmes: ambitions, difficultés et résultats », pp. 567-600, in : Bernstein S., Milza P., Bianco J.-L., dirs, François Mitterrand - Les années du changement (1981-1984), Paris, Perrin.

— 2003, « Histoire des femmes, histoire du genre et sexe du chercheur », pp. 7087, in : Laufer J., Marry C., Maruani M., dirs, Le travail du genre. Les sciences sociales à l'épreuve des différences de sexe, Paris, Éd. La Découverte-MAGE. 
— 2004a, « L'aventure intellectuelle de l'histoire des femmes en France », pp. 31 I-338, in : Racine N., Trebitsch M., dirs, Intellectuelles. Du genre en histoire des intellectuels, Bruxelles, Éd. Complexe.

— 2004b, « Femmes, féminisme, recherches : regard sur le bief 》, pp. 163-184, in : Bernos M., Bitton M., dirs, Femmes, Familles, Filiations, Mélanges en l'honneur d'Yvonne Knibiehler, Aix-en-Provence, Presses de l'université de Provence.

- 2005a, «Écrire l'histoire des femmes et du genre en France », Historiens\&Géographes, 392, pp. 71-81.

— 2005b, « Da Pénélope à Clio. Forze e debolezze della storia delle donne in Francia », pp. I63- I 78, in : Palazzi M., Porciani I., eds, Storiche di ieri e di oggi. Dalle autrici dell'Ottocento alle reviste di storia delle donne, Rome, Viella.

— 2007, Écrire l'histoire des femmes et du genre, Lyon, ENs Éd.

Thébaud Fr., Dermenjian G., 2009, Quand les femmes témoignent. Histoire, orale, histoire des femmes, mémoire des femmes, Paris, Éd. Publisud. 